\title{
Fabrication of small-diameter vascular scaffolds by heparin-bonded P(LLA-CL) composite nanofibers to improve graft patency
}

This article was published in the following Dove Press journal:

International Journal of Nanomedicine

6 June 2013

Number of times this article has been viewed

\section{Sheng Wang ${ }^{1, *}$ \\ Xiu $\mathrm{M} \mathrm{Mo}^{2, *}$ \\ Bo J Jiang' \\ Cheng J Gao' \\ Hong S Wang ${ }^{2}$ \\ Yu G Zhuang' \\ Li J Qiu ${ }^{2}$}

'Department of Emergency and Critical Care Medicine, Shanghai Tenth People's Hospital, Tongji University, Shanghai, People's Republic of China; ${ }^{2}$ State Key Laboratory for Modification of Chemical Fibers and Polymer Materials, Donghua University, Shanghai, People's Republic of China

*These authors contributed equally to this work
Correspondence: Sheng Wang Department of Emergency and Critical Care Medicine, Shanghai Tenth People's Hospital, Tongji University, Shanghai 200072, People's Republic of China

Tel +862166307153

Fax +862166303983

Email wangsheng@tongji.edu.cn
Abstract: The poor patency rate following small-diameter vascular grafting remains a major hurdle for the widespread clinical application of artificial blood vessels to date. Our previous studies found that electrospun poly(L-lactide-co-epsilon-caprolactone) (P[LLA-CL]) nanofibers facilitated the attachment and growth of endothelial cells (EC), and heparin incorporated into $\mathrm{P}($ LLA-CL) nanofibers was able to release in a controlled manner. Hence, we hypothesized that heparin-bonded P(LLA-CL) vascular scaffolds with autologous EC pre-endothelialization could significantly promote the graft patency rate. To construct a small-diameter vascular scaffold, the inner layer was fabricated by heparin-bonded P(LLA-CL) nanofibers through coaxial electrospinning, while the outer layer was woven by pure P(LLA-CL) nanofibers. Except dynamic compliance ( $5.4 \pm 1.7$ versus $\left.12.8 \pm 2.4 \times 10^{-4} / \mathrm{mmHg}, P<0.05\right)$, maximal tensile strength, burst pressure, and suture retention of the composite, scaffolds were comparable to those of canine femoral arteries. In vitro studies indicated that the scaffolds can continuously release heparin for at least 12 weeks and obtain desirable endothelialization through dynamic incubation, which was confirmed by EC viability and proliferation assay and scanning electronic microscopy. Furthermore, in vivo studies demonstrated that pre-endothelialization by autologous ECs provided a better effect on graft patency rate in comparison with heparin loading, and the united application of pre-endothelialization and heparin loading markedly promoted the 24 weeks patency rate of $\mathrm{P}$ (LLA-CL) scaffolds ( $88.9 \%$ versus $12.5 \%$ in the control group, $P<0.05)$ in the canine femoral artery replacement model. These results suggest that heparin-bonded P(LLA-CL) scaffolds have similar biomechanical properties to those of native arteries and possess a multiporous and biocompatible surface to achieve satisfactory endothelialization in vitro. Heparin-bonded P(LLA-CL) scaffolds with autologous EC pre-endothelialization have the potential to be substitutes for natural small-diameter vessels in planned vascular bypass surgery.

Keywords: electrospinning, heparin, vascular graft, endothelialization, patency rate

\section{Introduction}

Small-diameter vessels (inner diameter [ID] $<6 \mathrm{~mm}$ ) are extensively utilized to treat coronary and peripheral artery diseases. Although autologous veins or arteries are currently the preferred option, the availability of suitable native vessels is frequently in limited supply or restricted dimensions due to pre-existing vascular diseases or disease progression, ${ }^{1}$ thus there is an increasing demand for synthetic vascular grafts accompanied by climbing incidences of coronary and peripheral artery diseases. ${ }^{2}$ Unfortunately, owing to acute thrombosis and intimal hyperplasia, graft patency of synthetic conduits in small-diameter situations is disappointing so far, ${ }^{3}$ therefore, how to improve the long-term patency rate of small-diameter artificial blood vessels remains a major challenge and opportunity for vascular tissue engineering. ${ }^{2,4,5}$ 
In the native vasculature, an endothelial cell (EC) monolayer called the intima serves as a nonthrombogenic surface between circulating blood and the arterial wall through the expression of anticoagulant and fibrinolytic agents, also controlling platelet adhesion, aggregation, and activation important steps for blood coagulation. ${ }^{6}$ Additionally, the endothelium preserves the structural integrity of vessels to prevent inflammatory responses, which are closely linked to the occurrence of intimal hyperplasia. ${ }^{3}$ Hence, the coverage of intact and functional endothelium is a critical component of tissue-engineered blood vessels to maintain graft patency. Over the past decades, numerous efforts have been dedicated to achieve steady endothelialization of vascular scaffolds with moderate success, including the development of novel biocompatible materials, ${ }^{7}$ surface modification, ${ }^{6}$ innovative EC seeding techniques, or stem cell seeding. ${ }^{8}, 9$

Heparin is a polysaccharide anticoagulant with potent inhibitory effects on coagulation and a long history of safety and efficacy in the prevention and treatment of thrombosis. ${ }^{10}$ Moreover, medical devices coated by heparin, such as vascular grafts ${ }^{11}$ and coronary arterial stents, ${ }^{12}$ have successfully enhanced the patency rate and improved patient outcomes. Therefore, vascular scaffolds incorporated with heparin may further improve graft patency.

The progress of electrospinning technology has enabled the production of nanofiber-based porous scaffolds to facilitate cell attachment and tissue growth. ${ }^{13}$ Electrospinning can also create scaffolds to serve as controlled-release delivery devices. ${ }^{14}$ Indeed, our previous studies found that both smooth muscle cells (SMCs) and ECs adhered and proliferated well on electrospun poly(L-lactide-co-epsiloncaprolactone) (P[LLA-CL]) nanofibers. ${ }^{15,16}$ Recently, we successfully produced heparin-bonded P(LLA-CL) nanofibers through coaxial electrospinning to control the release of heparin. ${ }^{17}$ In this study, we intend to verify whether heparin-bonded P(LLA-CL) scaffolds facilitate the adhesion and growth of ECs, and whether the united application of pre-endothelialization by autologous ECs and the steady release of heparin from heparin-bonded P(LLA-CL) scaffolds significantly enhance graft patency rate in a canine femoral artery replacement model.

\section{Materials and methods}

All experimental procedures involving animals were in accordance with the institutional guidelines for animal care and approved by the Animal Ethics Committee of Shanghai Tenth People's Hospital, Shanghai, People's Republic of China.

\section{Scaffold fabrication}

Heparin-bonded P(LLA-CL) scaffolds were fabricated by a self-made coaxial electrospinning device, illustrated in Figure 1A. The inner layer of the scaffolds was composed of nanofibers through coaxial electrospinning, in which the core solution was $25 \%$ sodium heparin (13 kDa; Runjie Chemicals, Shanghai, People's Republic of China) and injected at a rate of $0.2 \mathrm{~mL} /$ hour and the shell solution $6 \% \mathrm{P}(\mathrm{LLA}-\mathrm{CL})$ (130 kDa; Sigma-Aldrich, St Louis, MO, USA) dissolved in 2, 2, 2-trifluoroethanol (Fine Chemicals, Shanghai, People's Republic of China) and fed at $0.8 \mathrm{~mL} /$ hour. The outer layer was woven by injecting the P(LLA-CL) solution at $1.0 \mathrm{~mL} /$ hour; this protocol was also used to construct pure $\mathrm{P}$ (LLA-CL) and polycaprolactone ([PCL] 70-90 kDa; Sigma-Aldrich) scaffolds. During electrospinning, room temperature was kept within $22^{\circ} \mathrm{C}-25^{\circ} \mathrm{C}$ with a relative humidity of $40 \%-50 \%$; a stainless steel mandrel (outer diameter $[\mathrm{OD}]=3 \mathrm{~mm}$ ) rotating at $250 \mathrm{rpm}$ was applied as the collecting rod. The distance between the sprayer tip and the mandrel was fixed to $15 \mathrm{~cm}$ and a positive voltage of $18 \mathrm{kV}$ was applied through a high-voltage generator. The scaffolds were dried under vacuum at room temperature for 48 hours before further characterization.

\section{Scaffold characterization}

The morphology of the scaffolds was observed by scanning electronic microscopy ([SEM] JSM-5600; JSM Ltd, Tokyo, Japan). Specimens were immersed into liquid nitrogen, then rapidly cut into $0.5 \mathrm{~cm}$-thick cross sections and imaged under SEM. Nanofiber diameter and layer thickness were measured by image analysis software (Image-J; National Institutes of Health [NIH], Bethesda, MD, USA).

To assess the mechanical properties of the scaffolds, canine femoral arteries were selected as the control. Tensile strength was measured by an Instron tensile tester (model 5544; Norwood, MA, USA). Specimens were cut into banded sections and incubated in phosphate-buffered saline ([PBS] $\mathrm{pH} 7.4$ ) in a $37^{\circ} \mathrm{C}$ shaker. Each specimen was mounted onto the clamps and pulled at $5 \mathrm{~mm} /$ minute crosshead speed until rupture, and maximal tensile strength was calculated by Bluehill software (Norwood, MA, USA). Burst pressure was measured by gradually increasing hydrostatic pressure within the scaffolds at a rate of $80 \mathrm{mmHg} /$ minute until rupture. To determine suture retention, a single throw of 5-0 prolene suture (Ethicon, Bridgewater, NJ, USA) was pulled ( $2 \mathrm{~mm} / \mathrm{second})$ through a scaffold and secured to the force transducer of the Instron testing device. Suture retention strength was defined as the maximal force recorded prior to pull-out of the suture. Dynamic compliance (C) of 

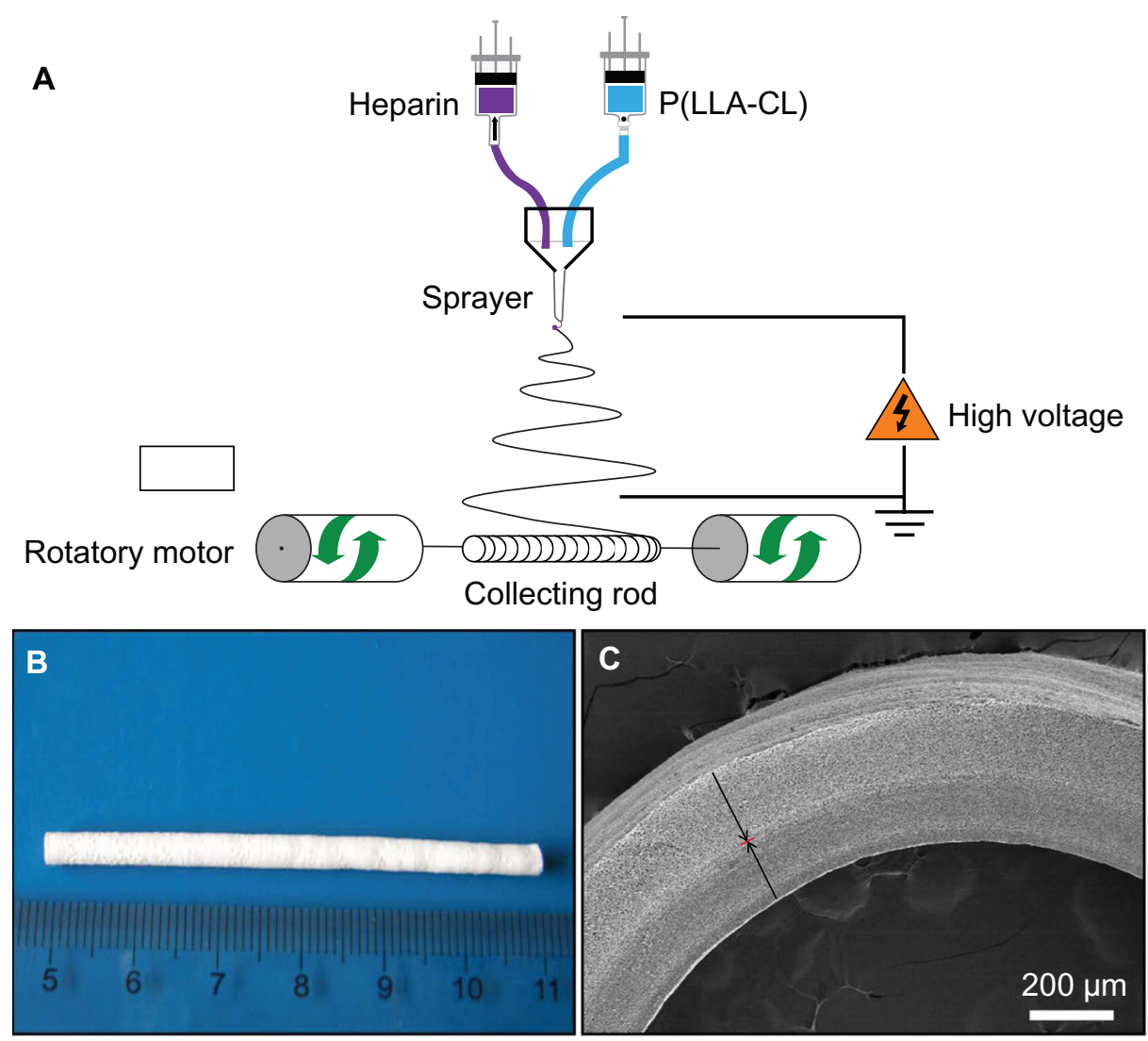

Figure I Fabrication of heparin-bonded poly(L-lactide-co-epsilon-caprolactone) (P[LLA-CL]) scaffolds.

Notes: (A) Schematic diagram of coaxial electrospinning device. (B) Typical heparin-bonded P(LLA-CL) tubular scaffold. (C) Cross sectional image of the heparin-bonded $\mathrm{P}(\mathrm{LLA}-\mathrm{CL})$ scaffold by scanning electronic microscopy at the magnification of $\times 50$. The red line between the two black arrows indicates the interface between the inner layer and the outer layer of the scaffold.

the scaffolds was determined by measuring the change in ID when the pressure $(\mathrm{P})$ was varied between 80 and $120 \mathrm{mmHg}$, and compliance was calculated by the following equation:

$$
\mathrm{C}=\frac{\left(\mathrm{ID}_{120}-\mathrm{ID}_{80}\right) / \mathrm{ID}_{80}}{\mathrm{P}_{120}-\mathrm{P}_{80}}
$$

\section{In vitro release of heparin from the scaffolds}

Heparin-bonded P(LLA-CL) scaffolds were cut into $5 \mathrm{~mm}$ thick rings and put into 12 -well plates one by one. After PBS $(5 \mathrm{~mL})$ was added into each well, the plates were sealed and stored in an humidified incubator at $37^{\circ} \mathrm{C}$ to prevent the evaporation of water. Following varying numbers of weeks of incubation, up to 12 weeks, $1.0 \mathrm{~mL}$ supernatant was acquired from each well and heparin concentration was assayed by toluidine blue method as described previously. ${ }^{17}$ Toluidine blue $(3.0 \mathrm{~mL})$ was added into each supernatant and reacted with heparin for 2 hours at $37^{\circ} \mathrm{C}$, then hexane $(3.0 \mathrm{~mL})$ was added and the sample solution stirred vigorously to separate the heparin-toluidine blue complex.
Samples were tested at $630 \mathrm{~nm}$ by a spectrophotometer (Agilent WFH-203B; PerkinElmer, Waltham, MA, USA).

\section{In vitro endothelialization of the scaffolds}

Autologous ECs were isolated from canine femoral veins by enzyme digestion. Briefly, both ends of the femoral vein were cannulated and all branches ligated. The vein was then washed thoroughly by PBS, filled with $0.25 \%$ trypsin solution, and digested for 6 minutes in a $\mathrm{CO}_{2}$ incubator $\left(37^{\circ} \mathrm{C}\right.$, $95 \%$ air $/ 5 \% \mathrm{CO}_{2}$ ). The EC suspension was transferred into a $15 \mathrm{~mL}$ centrifuge tube containing Dulbecco's modified Eagle's medium (Gibco; Life Technologies, Carlsbad, CA, USA) supplemented with $20 \%$ fetal bovine serum, $100 \mathrm{U} / \mathrm{mL}$ penicillin, and $100 \mathrm{mg} / \mathrm{mL}$ streptomycin. After the tubes were centrifuged at $600 \times g$ for 5 minutes, cell pellets were resuspended in the culture medium and ECs were purified by the preplating method $\left(2\right.$ hours, $\left.37^{\circ} \mathrm{C}\right)$ to remove fibroblasts; finally, unattached cells were transferred into culture flasks and cultured within the culture medium containing proper concentration of vascular endothelial growth factor (Gibco; Life Technologies) in the $\mathrm{CO}_{2}$ incubator. 
After 1 week of culture, the purity of ECs was determined by fluorescence microscopy (Figure 3B). Cultured ECs in the culture flasks were washed with PBS three times and immersed in a PBS solution containing 2\% paraformaldehyde for 10 minutes at room temperature. After rinsing with PBS again, the permeabilization solution $(0.2 \%$ Triton X-100 in PBS; Sigma-Aldrich) was added into the culture flasks for 10 minutes and rinsed again with fresh PBS. The ECs were then stained with diluted 4, 6-diamidino-2-phenylindole (Roche, Basel, Switzerland) and Alexa Fluor ${ }^{\circledR} 568$ Phalloidin (Molecular Probes; Life Technologies, NY, USA) overnight in a dark environment. After rinsing with PBS to remove the residual fluorescent dye, the sheets were imaged under confocal microscope (TCS SP5; Leica Microsystems, Wetzlar, Germany). Cultured ECs with purity more than 95\% were then seeded onto PCL, P(LLA-CL), and heparinbonded P(LLA-CL) scaffolds, respectively, through a culture system. Each scaffold was tightly embedded into a sterilized polypropylene tube (ID $=5 \mathrm{~mm}$ ) with one end sealed by a $220 \mathrm{~nm}$ filter. The tube was filled with ECs suspended in the culture medium at a density of $3 \times 10^{5}$ cells $/ \mathrm{mL}$ before the other end was sealed and the tube placed onto a roller mixer at a rotation speed of $5 \mathrm{rpm}$. The whole system was placed in the $\mathrm{CO}_{2}$ incubator and the culture medium was refreshed every 24 hours.

Following 7 days of incubation, a CellQuanti-Blue ${ }^{\mathrm{TM}}$ cell viability assay kit (BioAssay Systems, Hayward, CA, USA) was used to determine EC viability and proliferation, and PCL scaffolds were chosen as the control of P(LLA-CL) and heparin-bonded P(LLA-CL) scaffolds due to the poor biocompatibility of PCL. ${ }^{18}$ This assay is based on the fact that 3-(4,5-Dimethylthiazol-2-yl)-2,5-diphenyltetrazolium bromide (MTT) can be reduced to purple formazan in the mitochondria of living cells, and this colored solution can be quantified by a spectrophotometer with a certain wavelength; thus, the number of viable cells is directly related to the amount of MTT formazan formed and its absorbance. Briefly, cultured scaffolds were cut into $0.5 \mathrm{~mm}$ segments and transferred into black 96-well plates with $90 \mu \mathrm{L}$ of culture medium. After $10 \mu \mathrm{L}$ of the assay reagent was added per well, the plates were incubated for 2.5 hours at $37^{\circ} \mathrm{C}$, the absorbance at $570 \mathrm{~nm}$ was then measured on a fluorescence microplate reader (Infinite 200; Tecan, Männedorf, Switzerland). Meanwhile, SEM was applied to confirm in vitro endothelialization. To perform SEM, the sheets were fixed in $4 \%$ glutaraldehyde aqueous solution at $4{ }^{\circ} \mathrm{C}$ for 2 hours, dehydrated by graded concentrations of ethanol, coated with gold sputter, and observed under the SEM at the voltage of $15 \mathrm{kV}$.

\section{In vivo assessment of the scaffolds}

A bilateral femoral artery replacement model in Beagle dogs was applied to compare the patency rates among the following four groups: P(LLA-CL) scaffolds without pre-endothelialization; P(LLA-CL) scaffolds with pre-endothelialization; heparin-bonded P(LLA-CL) scaffolds without pre-endothelialization; and heparin-bonded P(LLACL) scaffolds with pre-endothelialization. All scaffolds had an ID of $4 \mathrm{~mm}$ and a length of 5-6 cm. Of a total of 20 elderly Beagle dogs (17.5-28 kg, age $>3$ years), ten animals were used to implant scaffolds without pre-endothelialization, and the remaining 10 animals were used to implant pre-endothelialized scaffolds into the corresponding donor of isolated autologous ECs. Anesthesia was maintained by intraperitoneal injection of amobarbital sodium $(20 \mathrm{mg} / \mathrm{kg})$, and both sides of the femoral artery were exposed. After $100 \mathrm{U} / \mathrm{kg}$ of sodium heparin was injected intravenously, one side of the femoral artery, approximately $5 \mathrm{~cm}$ in length, was excised when both ends of the artery were clamped, and a scaffold was implanted with 7-0 prolene sutures (Ethicon). A similar operation was completed to implant another scaffold into the other side of the artery. When no blood leakage was confirmed, by the reintroduction of blood flow, the incisions were stitched up with 4-0 silk sutures. Aspirin tablets (300 mg/day; Bayer AG, Leverkusen, Germany) were given orally from the day of surgery throughout the experimental procedure.

Color Doppler flow imaging (CDFI) was done repeatedly at 1,2,4, and 12 weeks after scaffold implantation to monitor graft patency. After 24 weeks of implantation, digital subtraction angiography (DSA) was performed to visualize the patency of the implanted scaffolds.

\section{Statistical analysis}

The results were analyzed by SigmaStat 3.5 (Systat Software, Point Richmond, CA, USA) and $P<0.05$ was considered statistically significant. Mechanical properties and EC viability were presented as mean \pm standard error and analyzed by Student's $t$-test, followed by a post hoc test when appropriate. Patency rates were presented as the percentages and the comparisons between groups were evaluated by z-test.

\section{Results Scaffold characterizations}

Heparin-bonded P(LLA-CL) scaffolds had an ID of $4 \mathrm{~mm}$ (Figure 1B) and consisted of two layers: the inner layer was $0.2 \mathrm{~mm}$ in thickness, fabricated by nanofibers containing both heparin and P(LLA-CL), while the outer layer was composed by P(LLA-CL) fibers only, with a thickness of 
$0.3 \mathrm{~mm}$ (Figure 1C). As illustrated in Figure 2, the ODs of P(LLA-CL) and heparin-bonded P(LLA-CL) nanofibers were several hundred nanometers, these nanofibers comprising a multiporous structure with a large surface area. Pore size ranged from hundreds of nanometers to several microns.

The mechanical properties of the scaffolds are summarized in Table 1. Maximal tensile strength, burst pressure, and suture retention of $\mathrm{P}(\mathrm{LLA}-\mathrm{CL})$ scaffolds were comparable to those of canine femoral arteries; nevertheless, scaffold compliance showed a statistical difference $(3.7 \pm 1.2$ versus $\left.12.8 \pm 2.410^{-4} / \mathrm{mmHg}, P<0.05\right)$. The embedding of heparin tended to reduce maximal tensile strength and burst pressure and increase suture retention and compliance in comparison to those in P(LLA-CL) scaffolds, but without statistical difference. The compliance in heparin-bonded P(LLA-CL) scaffolds was also significantly decreased as compared to native arteries $\left(5.4 \pm 1.7\right.$ versus $12.8 \pm 2.410^{-4} / \mathrm{mmHg}$, $P<0.05)$.

\section{In vitro release of heparin from the scaffolds}

The controlled release of heparin from heparin-bonded $\mathrm{P}$ (LLA-CL) scaffolds was validated in vitro. As shown in Figure 3A, about one-quarter of heparin was released from the scaffolds into PBS solution within the first week, after which there was a sustained release of heparin throughout the 12-week observation period, over which the heparinrelease curve ascended gradually. By the end of 12 weeks, the percentage of heparin released from the scaffolds reached more than $90 \%$.

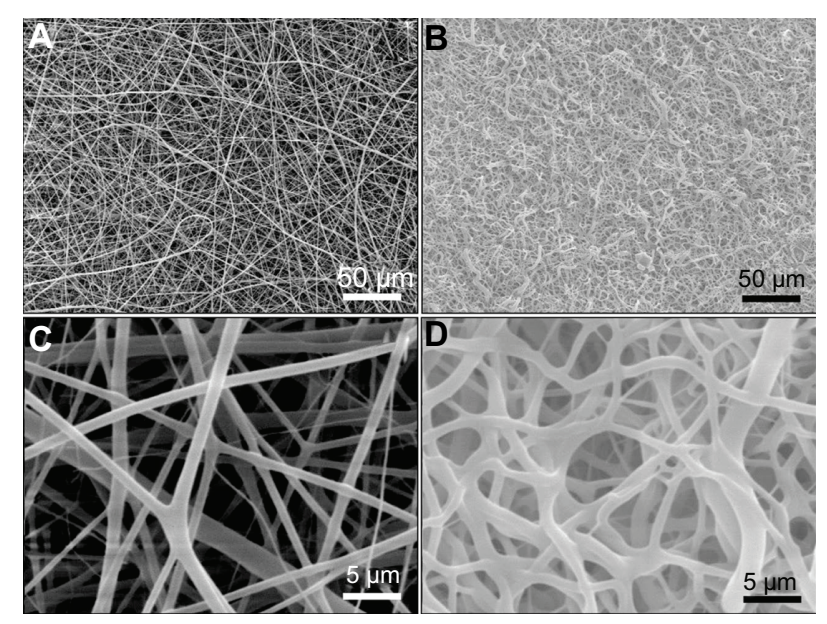

Figure 2 Electron microscope scanning of poly(L-lactide-co-epsilon-caprolactone) $(P[L L A-C L])$ nanofibers and heparin-bonded $P(L L A-C L)$ nanofibers.

Notes: (A) Nanofiber morphology of P(LLA-CL) and (B) heparin-bonded P(LLA$\mathrm{CL})$ nanofibers at low $(\times 500)$ magnification. (C) Nanofiber morphology of P(LLA$\mathrm{CL})$ and $(\mathrm{D})$ heparin-bonded $\mathrm{P}(\mathrm{LLA}-\mathrm{CL})$ nanofibers at high $(\times 5000)$ magnification.
Table I Mechanical properties of the tubular scaffolds

\begin{tabular}{lccc}
\hline & CFA & PLC & HPLC \\
\hline Tensile strength (Mpa) & $2.4 \pm 0.6$ & $3.8 \pm 0.9$ & $2.9 \pm 0.4$ \\
Burst pressure (mmHg) & $2148 \pm 264$ & $3175 \pm 438$ & $2840 \pm 354$ \\
Suture retention (Newton) & $5.4 \pm 1.1$ & $4.3 \pm 0.8$ & $4.6 \pm 1.4$ \\
Compliance $\left(10^{-4} / \mathrm{mmHg}\right)$ & $12.8 \pm 2.4$ & $3.7 \pm 1.2^{*}$ & $5.4 \pm 1.7^{*}$ \\
\hline
\end{tabular}

Notes: All values are mean \pm standard error $(n=6), * P<0.05$ versus canine femoral arteries.

Abbreviations: CFA, canine femoral arteries; PLC, poly(L-lactide-co-epsiloncaprolactone) (P[LLA-CL]) scaffolds; HPLC, heparin-bonded P(LLA-CL) scaffolds.

\section{In vitro endothelialization of the scaffolds}

After 1 week of dynamic incubation, CellQuanti-Blue ${ }^{\mathrm{TM}}$ assay indicated that EC viability and proliferation was significantly higher in the ECs seeded onto P(LLA-CL) and heparin-bonded P(LLA-CL) scaffolds than in those seeded on PCL scaffolds (Figure 4A). Moreover, SEM showed that almost no ECs were attached onto the lumen of PCL scaffolds following 1 week of dynamic culture (Figure 4B), whereas lots of ECs were adhered and grown on the inner surface of P(LLA-CL) scaffolds (Figure 4C) and a well-spread monolayer of ECs was found on the luminal surface of heparinbonded P(LLA-CL) scaffolds (Figure 4D).

\section{In vivo assessment of the scaffolds}

Of the 20 Beagle dogs on which femoral artery grafting was performed (Figure 5A), two animals used to implant scaffolds without pre-endothelialization were excluded due to surgical-related acute bleeding, and another animal used to implant pre-endothelialized scaffolds was also excluded due to severe infection of the incisional wound caused by improper asepsis. Although seven of eight femoral arteries replaced by P(LLA-CL) scaffolds without pre-endothelialization remained unblocked 1 week after implantation by CDFI (Figure 5B), the patency rate was rapidly reduced to less than $15 \%(1 / 8)$ 24 weeks after implantation by DSA assessment (Figure 5C).
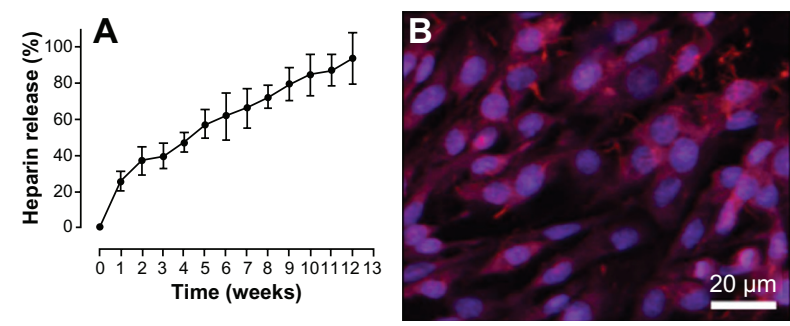

Figure 3 (A) Controlled release of heparin from heparin-bonded poly(L-lactide-coepsilon-caprolactone) (P[LLA-CL]) scaffolds in vitro. (B) Representative photograph to determine the purity of cultured endothelial cells by fluorescent microscopy at the magnification of $\times 200$.

Notes: The percentage of heparin release from the scaffolds was determined every week and this process lasted at least 12 weeks. All data are expressed as mean \pm standard error $(n=6)$. 

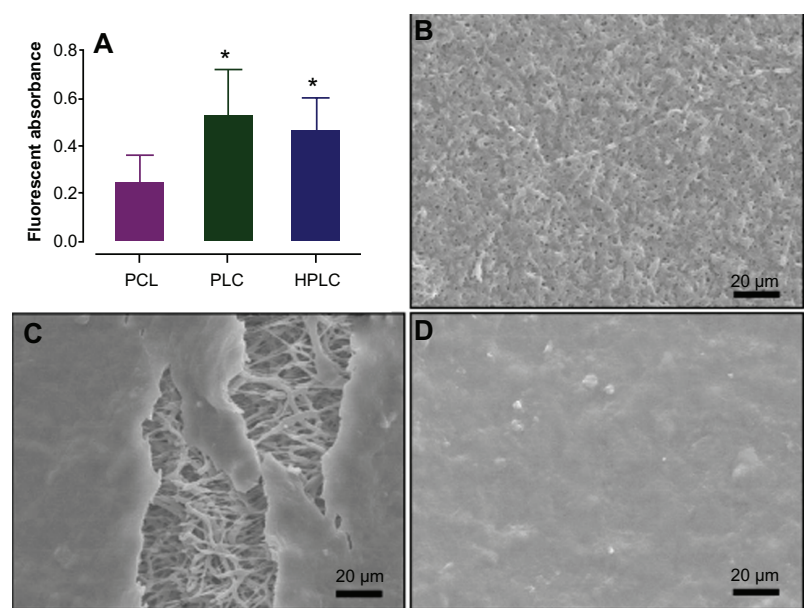

Figure 4 The endothelialization of heparin-bonded poly(L-lactide-co-epsiloncaprolactone) (P[LLA-CL]) scaffolds in vitro.

Notes: (A) Endothelial cell viability on PCL, P(LLA-CL), and heparin-bonded P(LLA$\mathrm{CL}$ ) scaffolds after I week of dynamic culture. (B-D) Representative images of the endothelialization of $P C L, P(L L A-C L)$, and heparin-bonded $P(L L A-C L)$ scaffolds by scanning electronic microscopy at the magnification of $\times 1000$, respectively. Endothelial cell viability and proliferation was presented as the fluorescent absorbance determined by CellQuanti-Blue ${ }^{\mathrm{TM}}$ assay. All values are illustrated as mean \pm standard error $(n=8), * P<0.05$ versus $P C L$ scaffolds.

Abbreviations: HPLC, heparin-bonded P(LLA-CL) scaffolds; PCL, polycaprolactone scaffolds; PLC, P(LLA-CL) scaffolds.

Both autologous EC pre-endothelialization and heparin loading significantly improved the 24-week patency rates of $\mathrm{P}($ LLA-CL) scaffolds in comparison with those of P(LLA-CL) scaffolds without pre-endothelialization (Figure 5D), but the effect of pre-endothelialization on patency rate was much better than heparin loading (66.7\% versus $37.5 \%)$. The united application of pre-endothelialization and heparin loading markedly boosted the patency rate to more than $85 \%$ after 24 weeks of implantation, which was verified by completely full of contrast medium throughout the scaffolds in the DSA images (Figure 5C).

\section{Discussion}

To date, the poor patency rate following small-diameter vascular grafting remains a major hurdle for the widespread clinical application of synthetic conduits. In the present study, we demonstrated that the small-diameter scaffolds, fabricated by heparin-bonded P(LLA-CL) nanofibers through coaxial electrospinning in the inner layer, possessed mechanical properties comparable to those of canine femoral arteries and had a porous and biocompatible surface that facilitated the adhesion and growth of ECs. More importantly, pre-endothelialization of heparin-bonded P(LLA-CL) scaffolds by autologous ECs, combined by the sustained release of heparin from the scaffolds, markedly improved the patency rate 24 weeks after implantation in the canine femoral artery replacement model.

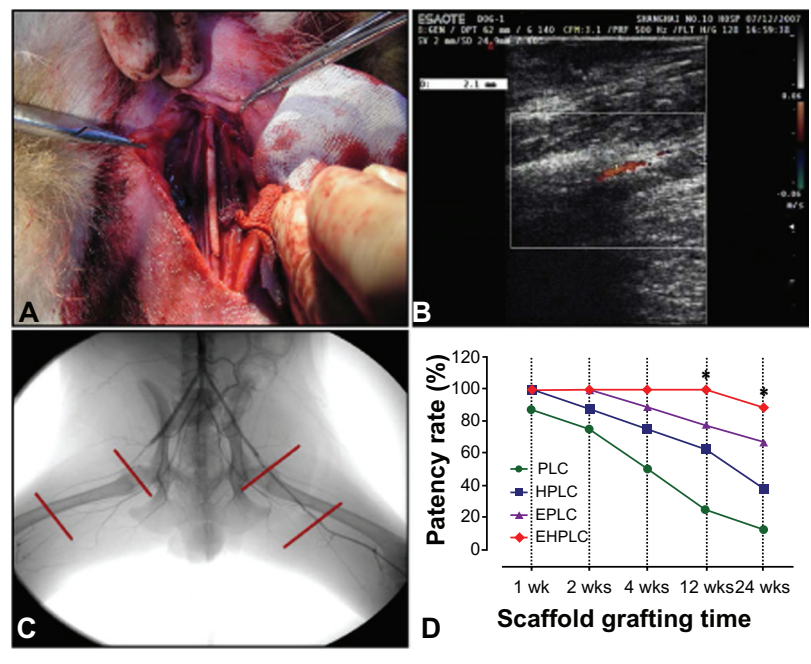

Figure 5 Assessments of the implanted scaffolds 24 weeks after vascular grafting. Notes: (A) Surgical implantation of a scaffold into a femoral artery in an elderly Beagle dog. (B) Monitoring the patency of the implanted scaffold by color Doppler flow imaging. (C) Digital subtraction angiography of the grafted scaffolds. The red lines indicate the location of vascular grafts on both sides of canine femoral arteries. The scaffold implanted into the left femoral artery was patent and the other one was totally blocked. (D) Patency rates of various scaffolds after I, 2, 4, I2, and 24 weeks of surgical implantation. ${ }^{*} P<0.05$ versus poly(L-lactide-co-epsilon-caprolactone) (P[LLA-CL]) scaffolds without pre-endothelialization.

Abbreviations: EHPLC, heparin-bonded $P(L L A-C L)$ scaffolds with preendothelialization; EPLC, P(LLA-CL) scaffolds with pre-endothelialization; HPLC, heparin-bonded P(LLA-CL) scaffolds without pre-endothelialization; PLC, P(LLA$\mathrm{CL})$ scaffolds without pre-endothelialization.

Poly-L-lactide (PLLA) and PCL were chosen to construct the scaffolds in the current study, as the former has been shown to possess excellent biocompatibility and promote the attachment and sustained proliferation of ECs, ${ }^{19}$ while the latter has tunable elasticity and tensile strength that are crucial for vascular tissue engineering. ${ }^{20}$ Furthermore, the degradation rate of these two polymers is slow, which is essential for the design of long-term implantable scaffolds. ${ }^{21}$ The united application of PLLA and PCL is based on the fact that one polymer is often not enough to meet all the requirements of prosthetic vascular grafts. ${ }^{22}$ For example, the biocompatibility of PCL is poor owing to its hydrophobicity and lack of cellular specific interaction, ${ }^{18}$ while PLLA has favorable biocompatibility. ${ }^{19}$ Moreover, we have previously reported that electrospun P(LLA-CL) nanofibers were able to secure the adhesion and growth of ECs and SMCs; ${ }^{15,16}$ this copolymer has also been suggested as an ideal material for vascular tissue engineering.?

The mechanical properties of heparin-bonded P(LLA-CL) scaffolds, including maximal tensile strength, burst pressure, and suture retention, were comparable to those of canine femoral arteries, while dynamic compliance was not as good as that of native arteries, suggesting that the mechanical properties of the scaffolds were within the physiological 
range of natural small-diameter arteries in general, although their dynamic compliance required further improvement. These biomechanical properties not only guaranteed the structural integrity of vascular scaffolds, but also protected heparin-bonded P(LLA-CL) nanofibers from breaking into segments under pressure, thus ensuring the sustained release of heparin. The SEM results indicated that heparin-bonded P(LLA-CL) scaffolds were woven by nanoscale fibers with a porous structure at submicron level and a large surface area microstructural characteristics that are crucial for cell seeding and growth on the luminal surface of the scaffolds. ${ }^{13}$

In this study, SEM study indicated that the luminal surface of heparin-bonded P(LLA-CL) scaffolds was covered by a well-spread EC monolayer after 1 week of dynamic incubation in vitro, although almost no ECs were attached to the inner layer of PCL scaffolds, suggesting that heparinbonded P(LLA-CL) was a much more biocompatible material for EC seeding and proliferation than PCL. This was further confirmed by CellQuanti-Blue ${ }^{\mathrm{TM}}$ assay, in which autologous ECs seeded onto heparin-bonded P(LLA-CL) scaffolds had significantly higher EC viability and proliferation in comparison with those seeded on PCL scaffolds following 1 week of pre-endothelialization.

It has been previously reported that heparin is a potent antiproliferative agent $;{ }^{10}$ interestingly, the extent of EC proliferation was no different between P(LLA-CL) and heparinbonded P(LLA-CL) scaffolds after 1 week of dynamic culture in our study. The causes of this are unknown, but may be explained by the controlled release of heparin, which maintained a relatively low concentration of heparin and prevented heparin from exerting antiproliferative action.

It is well known that the anatomical and physiological properties of small-diameter arteries in large animals are similar to those of human beings, ${ }^{23}$ thus large-animal models are the ideal choice by which to obtain important preclinical evidence. Moreover, the bilateral femoral artery replacement model provided us an extremely convenient and visualized comparison of patency rate. Previous studies reported that animals such as dogs owned the inherent ability to selfendothelialize the implanted vascular grafts, ${ }^{24}$ which would inevitably confound the effects of pre-endothelialization by autologous EC, hence, elderly animals were chosen to eliminate this defect in this study. Unfortunately, we did not compare the different extents of self-endothelialization between younger and elderly Beagle dogs in the current report, but the application of elderly animals may better mimic the aged population who require small-diameter vascular grafting in clinical situations.
Despite the application of anticoagulant aspirin in our study, the 24 -week patency rate was only $12.5 \%$ when P(LLA-CL) scaffolds without pre-endothelialization were implanted into canine femoral arteries. Except for the use of aged animals to prevent self-endothelialization, we believe that the lack of EC coverage was the major cause, as the 24-week patency rate rose to $66.7 \%$ when P(LLA-CL) scaffolds with pre-endothelialization were used. Although the application of heparin-bonded P(LLA-CL) scaffolds also improved the 24-week patency rate compared to that of P(LLA-CL) scaffolds without pre-endothelialization (37.5\% versus $12.5 \%$ ), the level of increase was not as good as that of pre-endothelialization ( $37.5 \%$ versus $66.7 \%$ ), suggesting that pre-endothelialization of P(LLA-CL) scaffolds by autologous ECs has a better effect on patency rate than heparin loading. Furthermore, more than $90 \%$ of the bonded heparin was released after 12 weeks in the in vitro study, suggesting that almost no heparin was available from 12 to 24 weeks after heparin-bonded P(LLA-CL) scaffold grafting, which may also contribute to the relatively lower increase of patency rate by heparin loading. More importantly, the united application of pre-endothelialization and heparin loading further improved the 24-week patency rate, which was more than $85 \%$ when femoral arteries were replaced by heparin-bonded P(LLA-CL) scaffolds with pre-endothelialization.

Several mechanisms may be responsible for the marked increase in patency rate: firstly, the coverage of autologous ECs through in vitro pre-endothelialization provided an anticoagulant and antithrombogenic luminal surface for the scaffolds to avoid acute thrombosis; ;,6 secondly, the coverage of ECs ensured the luminal integrity of the vascular grafts and prevented the occurrence of inflammatory responses, which have been demonstrated to cause intimal hyperplasia; ${ }^{25}$ thirdly, the sustained release of heparin from heparin-bonded P(LLA-CL) nanofibers reduced thrombinmediated fibrin formation and platelet activation. ${ }^{26} \mathrm{In}$ addition, heparin also offered a negative-charged surface to block the interaction between platelets and scaffolds by electrostatic repulsion, ${ }^{10}$ thus contributing to the prevention of thrombotic occlusion.

Although heparin-incorporated P(LLA-CL) scaffolds together with autologous EC pre-endothelialization may be a promising strategy to overcome the poor patency rate in small-diameter vascular grafting, the whole process to construct such vascular scaffolds required at least 2 weeks before they were suitable for implantation, indicating that these scaffolds can only be used in elective vascular surgery. Moreover, there were several limitations of the present study. Firstly, we did 
not investigate whether the scaffolds were still fully covered by autologous ECs in vivo after 24 weeks of implantation, thus the actual role of pre-endothelialization on patency rate remained to be confirmed. Secondly, intimal hyperplasia is an important cause of poor patency rate in small-diameter vascular grafting, particularly in the anastomotic area; ${ }^{27}$ we did not, however, evaluate intimal hyperplasia after scaffold implantation. Besides this, both PLLA and PCL are known to be biodegradable materials, ${ }^{20}$ unfortunately, the extent of scaffold degrading and the alteration of mechanical properties following 24 weeks of implantation were not determined by us. Finally, natural vessels are far more than pipes or tubes in the vascular system, ${ }^{28}$ but the physiological properties of implanted scaffolds, such as vasomotor function, were also not evaluated. Therefore, further studies are needed to solve these problems.

\section{Conclusion}

Heparin-bonded P(LLA-CL) scaffolds possessed similar biomechanical properties to those of native arteries, had a multiporous and biocompatible surface by which to achieve satisfactory endothelialization in vitro, and released heparin sustainably throughout the 12-week observation period. Preendothelialization by autologous ECs provided better effect on graft patency rate than heparin loading, and the united application of pre-endothelialization and heparin loading markedly promoted the 24 weeks patency rate of $P(L L A-C L)$ scaffolds in the femoral artery replacement model in elderly Beagle dogs. These results suggest that the controlled release of heparin from the scaffolds, along with autologous EC preendothelialization, could be a promising strategy by which to reverse the disappointing performance of prosthetic blood vessels in small-diameter vascular grafting.

\section{Acknowledgments}

This study was supported by National 863 High Technology Plan of China (2008AA03Z305), National Natural Science Foundation of China (31070871), Science and Technology Commission of Shanghai Municipality Program (11nm0506200), and the Reserve Academic Leader Program of Shanghai Tenth People's Hospital, Shanghai, People's Republic of China.

\section{Disclosure}

The authors report no conflicts of interest in this work.

\section{References}

1. McBane JE, Sharifpoor S, Labow RS, Ruel M, Suuronen EJ, Santerre JP. Tissue engineering a small diameter vessel substitute: engineering constructs with select biomaterials and cells. Curr Vasc Pharmacol. 2012; 10(3):347-360.
2. Peck M, Gebhart D, Dusserre N, McAllister TN, L'Heureux N. The evolution of vascular tissue engineering and current state of the art. Cells Tissues Organs. 2012;195(1-2):144-158.

3. Li S, Henry JJ. Nonthrombogenic approaches to cardiovascular bioengineering. Annu Rev Biomed Eng. 2011;13:451-475.

4. Chlupác J, Filová E, Bacáková L. Blood vessel replacement: 50 years of development and tissue engineering paradigms in vascular surgery. Physiol Res. 2009;58 Suppl 2:S119-S139.

5. Song Y, Feijen J, Grijpma DW, Poot AA. Tissue engineering of smalldiameter vascular grafts: a literature review. Clin Hemorheol Microcirc. 2011;49(1-4):357-374.

6. Khan OF, Sefton MV. Endothelialized biomaterials for tissue engineering applications in vivo. Trends Biotechnol. 2011;29(8):379-387.

7. Hung HS, Chen HC, Tsai CH, Lin SZ. Novel approach by nanobiomaterials in vascular tissue engineering. Cell Transplant. 2011;20(1): $63-70$.

8. Villalona GA, Udelsman B, Duncan DR, et al. Cell-seeding techniques in vascular tissue engineering. Tissue Eng Part B Rev. 2010;16(3): 341-350.

9. Krawiec JT, Vorp DA. Adult stem cell-based tissue engineered blood vessels: a review. Biomaterials. 2012;33(12):3388-3400.

10. Gray E, Hogwood J, Mulloy B. The anticoagulant and antithrombotic mechanisms of heparin. Handb Exp Pharmacol. 2012;(207):43-61.

11. Daenens K, Schepers S, Fourneau I, Houthoofd S, Nevelsteen A. Heparin-bonded ePTFE grafts compared with vein grafts in femoropopliteal and femorocrural bypasses: 1- and 2-year results. JVasc Surg. 2009;49(5):1210-1216.

12. Haude M, Konorza TF, Kalnins U, et al; Heparin-COAted STents in small coronary arteries Trial Investigators. Heparin-coated stent placement for the treatment of stenoses in small coronary arteries of symptomatic patients. Circulation. 2003;107(9):1265-1270.

13. Rathore A, Cleary M, Naito Y, Rocco K, Breuer C. Development of tissue engineered vascular grafts and application of nanomedicine. Wiley Interdiscip Rev Nanomed Nanobiotechnol. 2012;4(3):257-272.

14. Spadaccio C, Chello M, Trombetta M, Rainer A, Toyoda Y, Genovese JA. Drug releasing systems in cardiovascular tissue engineering. J Cell Mol Med. 2009;13(3):422-439.

15. Mo XM, Xu CY, Kotaki M, Ramakrishna S. Electrospun P(LLA-CL) nanofiber: a biomimetic extracellular matrix for smooth muscle cell and endothelial cell proliferation. Biomaterials. 2004;25(10):1883-1890.

16. Zhang K, Wang H, Huang C, Su Y, Mo X, Ikada Y. Fabrication of silk fibroin blended P(LLA-CL) nanofibrous scaffolds for tissue engineering. J Biomed Mater Res A. 2010;93(3):984-993.

17. Su Y, Li X, Liu Y, Su Q, Qiang ML, Mo X. Encapsulation and controlled release of heparin from electrospun poly(L-Lactide-coepsilon-Caprolactone) nanofibers. J Biomater Sci Polym Ed. 2011;22: 165-177.

18. Hiep NT, Lee BT. Electro-spinning of PLGA/PCL blends for tissue engineering and their biocompatibility. J Mater Sci Mater Med. 2010;21(6):1969-1978.

19. Lu H, Feng Z, Gu Z, Liu C. Growth of outgrowth endothelial cells on aligned PLLA nanofibrous scaffolds. J Mater Sci Mater Med. 2009;20(9):1937-1944.

20. Saik JE, McHale MK, West JL. Biofunctional materials for directing vascular development. Curr Vasc Pharmacol. 2012;10(3):331-341.

21. Naito Y, Shinoka T, Duncan D, et al. Vascular tissue engineering: towards the next generation vascular grafts. Adv Drug Deliv Rev. 2011;63(4-5):312-323.

22. Gui L, Zhao L, Spencer RW, et al. Development of novel biodegradable polymer scaffolds for vascular tissue engineering. Tissue Eng Part A. 2011;17(9-10):1191-1200.

23. Byrom MJ, Bannon PG, White GH, Ng MK. Animal models for the assessment of novel vascular conduits. J Vasc Surg. 2010;52(1): 176-195.

24. Seifalian AM, Tiwari A, Hamilton G, Salacinski HJ. Improving the clinical patency of prosthetic vascular and coronary bypass grafts: the role of seeding and tissue engineering. Artif Organs. 2002;26(4):307-320. 
25. Dahl SL, Blum JL, Niklason LE. Bioengineered vascular grafts: can we make them off-the-shelf? Trends Cardiovasc Med. 2011;21(3): 83-89.

26. Tatterton M, Wilshaw SP, Ingham E, Homer-Vanniasinkam S. The use of antithrombotic therapies in reducing synthetic small-diameter vascular graft thrombosis. Vasc Endovascular Surg. 2012;46(3): 212-222.
27. Bosiers M, Deloose K, Verbist J, et al. Heparin-bonded expanded polytetrafluoroethylene vascular graft for femoropopliteal and femorocrural bypass grafting: 1-year results. JVasc Surg. 2006;43(2):313-318; discussion 318-319.

28. Scharn DM, Daamen WF, van Kuppevelt TH, van der Vliet JA. Biological mechanisms influencing prosthetic bypass graft patency: possible targets for modern graft design. Eur J Vasc Endovasc Surg. 2012;43(1):66-72.
International Journal of Nanomedicine

\section{Publish your work in this journal}

The International Journal of Nanomedicine is an international, peerreviewed journal focusing on the application of nanotechnology in diagnostics, therapeutics, and drug delivery systems throughout the biomedical field. This journal is indexed on PubMed Central, MedLine, CAS, SciSearch $®$, Current Contents $® /$ Clinical Medicine,

\section{Dovepress}

Journal Citation Reports/Science Edition, EMBase, Scopus and the Elsevier Bibliographic databases. The manuscript management system is completely online and includes a very quick and fair peer-review system, which is all easy to use. Visit http://www.dovepress.com/ testimonials.php to read real quotes from published authors.

Submit your manuscript here: http://www.dovepress.com/international-journal-of-nanomedicine-journal 